\title{
étude sur maquette des vibrations dues au tir en souterrain
}

\author{
par \\ Ph. Weber \\ P. Saint Lot \\ École Nationale Supérieure des Techniques Industrielles et des Mines, Alès
}

RESUME - Le creusement à l'explosif du second tube d'un tunnel autoroutier à deux tubes induit des vibrations dont l'amplitude risque de provoquer, au niveau de l'ouvrage antérieur, une nuisance pouvant conduire à l'arrêt du trafic durant les travaux d'excavation.

Une étude sur maquette de laboratoire a été réalisée à partir de la configuration du tunnel de Ricard (Autoroute A8, travaux de contournement de Nice-Nord).

Après un examen des conditions de similitude du problème, l'étude a permis de dégager un certain nombre de résultats concernant la propagation et l'amortissement des vibrations de tir au voisinage du tube antérieur, lors de l'excavation du tube postérieur.

Une corrélation avec des mesures effectuées sur le site confirme la validité du modèle.

\section{INTRODUCTION}

Des travaux de doublement de tunnels autoroutiers en service sont actuellement en cours sur 1'autoroute A8, entre Nice et Menton. Des travaux de même nature seront ultérieurement effectués sur d'autres tronçons du réseau autoroutier national (autoroutes alpines) . Parmi les questions posées par ces travaux de doublement, figure notamment la suivante : est-il possible de poursuivre les travaux d'excavation sans interrompre le trafic dans le tunnel déjà en service? Pour pouvoir apporter des éléments de réponse à la question, il est nécessaire de définir les modes de propagation des vibrations induites le long du tube en service par les tirs de creusement.

La complexité géométrique du problème rend illusoire toute approche analytique ou numérique. L'approche retenue dans le cadre de cette étude, est donc celle d'un modèle réduit de laboratoire reproduisant, à

l'échelle de $1 / 100$ è, la géométrie du site.
Des mesures de vibrations effectuées par ailleurs par le CETu et le CETE, d'Aix-enProvence de juillet à septembre 1978 dans le tube Nord du tunnel de Ricard (autoroute A8, section La Turbie-Roquebrune) lors du creusement du tube sud, ont permis de confirmer, du moins partiellement, la validité de l'étude sur maquette.

\section{CARACTERISTIQUES DU MODELE EXPERIMENTAL}

1) La maquette

La maquette reproduit, à l'échelle de $1 / 100$ , un tronçon de tunnel de $100 \mathrm{~m}$ de longueur dans lequel les deux tubes sont parallèles et distants de $9,50 \mathrm{~m}$ (distance entre piedroits adjacents). La largeur de chaque tube est de $12 \mathrm{~m}$.

La maquette se compose d'un bloc en béton de chaux de forme parallélépipédique dans laquelle le tube Nord - en service - traverse le bloc de part en part, et le tube Sud - en cours de creusement - s'arrête 
à mi-hauteur, (fig.1).

Les tirs de creusement sont simulés par l'impact mécanique d'une bille en acier sur le front de taille du tube en cours de creusement.

Les caractéristiques du projectile et de l'impact (masse et vitesse d'impact) sont définies à partir de conditions de similitude.

2) Validité du modèle. Conditions de similitude

Les conditions de similitude déduites des équations de I'élastodynamique (MANDEL), imposent que la longueur d'onde de la vibration soit "à 1'échelle" des dimensions linéaires de l'ouvrage :

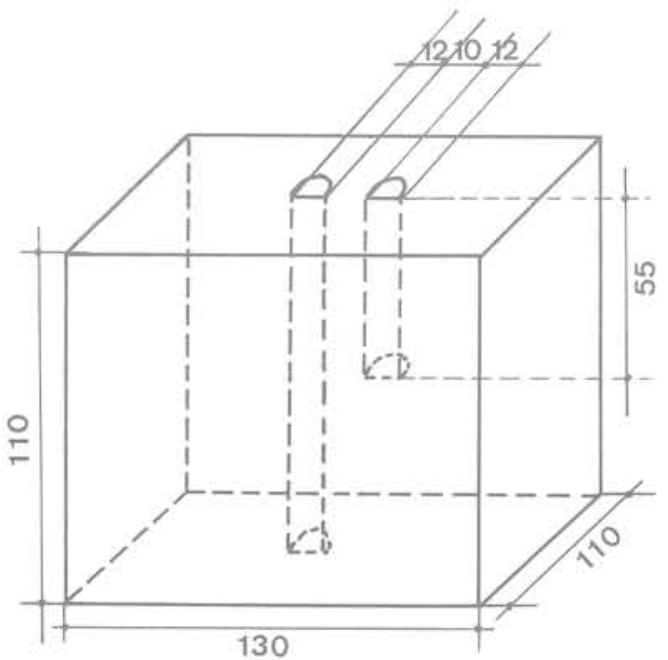

Fig.1 - Schéma de la maquette

$$
\frac{\lambda_{m}}{D_{m}}=\frac{\lambda_{s}}{D_{s}}
$$

où $\lambda_{m}$ et $D_{m}\left(\lambda_{S}\right.$ et $\left.D_{S}\right)$ désignent respectivement la longueur d'onde et le diamètre du tube (m: maquette, s : site).

Désignant par $C_{m}$ et $F_{m}\left(C_{s}\right.$ et $\left.F_{S}\right)$ la célérité des ondes acoustiques et la fréquence de la vibration, l'équation (1) devient :

$$
F_{m}=F_{s} \cdot \frac{C_{m}}{C_{s}} \cdot \frac{D_{s}}{D_{m}} \text {, }
$$

équation qui définit la valeur de la fréquence qui doit être générée par l'impact sur la maquette.
Avec $C_{m}=3200 \mathrm{~m} / \mathrm{s}, C_{\mathrm{s}}=4000 \mathrm{~m} / \mathrm{s}$, $\mathrm{D}_{\mathrm{s}} / \mathrm{D}_{\mathrm{m}}=100$ (échelle de la maquette) et $\mathrm{F}_{\mathrm{S}}=70$ Hertz (valeur modale correspondant aux mesures effectuées sur le site), $\mathrm{F}_{\mathrm{m}}=5600$ Hertz.

Par essais successifs, il est ainsi possible de définir les conditions d'impact diamètre et hauteur de chute du projectileassurant une fréquence de vibration respectant l'équation (2).

L'équation (2) ne préjuge en rien des intensitếs relatives de la vibration sur la maquette et sur le site; pour les définir, introduisons le rapport $\mathrm{K}$ des vitesses particulaires $v$ de la vibration aux points homologues des deux structures :

$$
\frac{\mathrm{V}_{\mathrm{m}}}{\mathrm{V}_{\mathrm{S}}}=\mathrm{K}
$$

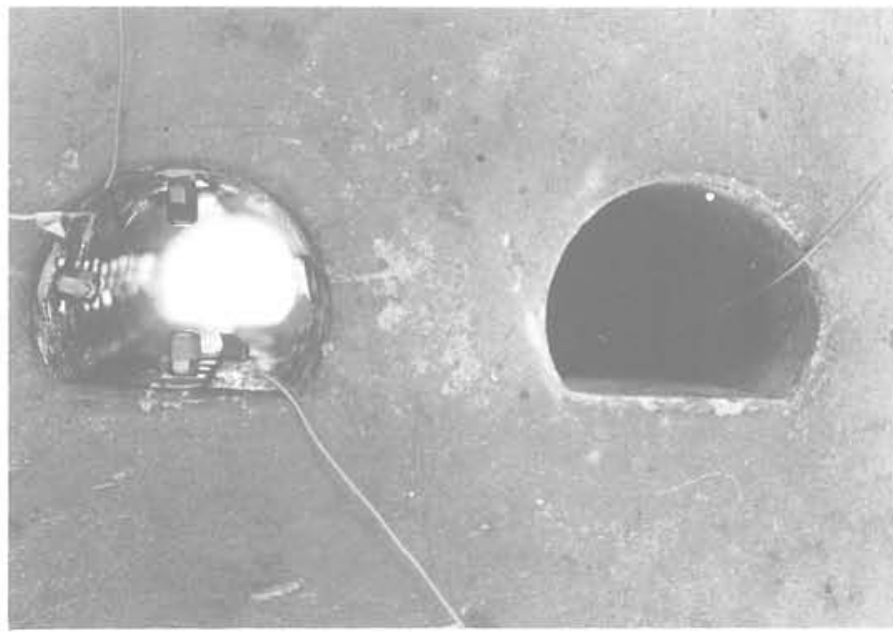

Fig.2 - Détail des deux tubes : à gauche, tube en service avec section équipée de 3 accéléromètres ; à droite, tube en cours de creusement.

Dans ces conditions, on établit que :

$$
\begin{aligned}
& \frac{\mathrm{u}_{\mathrm{m}}}{\mathrm{u}_{\mathrm{S}}}=\mathrm{K} \cdot \frac{\mathrm{F}_{\mathrm{S}}}{\mathrm{F}_{\mathrm{m}}} \quad\left(\mathrm{u}: \begin{array}{c}
\text { déplacement parti- } \\
\text { culaire }
\end{array}\right. \\
& \frac{\gamma_{\mathrm{m}}}{\gamma_{\mathrm{S}}}=\mathrm{K} \frac{\mathrm{F}_{\mathrm{m}}}{\mathrm{F}_{\mathrm{S}}}\left(\gamma: \begin{array}{l}
\text { accélération parti- } \\
\text { culaire })
\end{array}\right.
\end{aligned}
$$

Le rapport $\mathrm{K}$ définit ainsi les intensités relatives de la vibration.

\section{3) Protocole expérimental}

L'expérimentation met en jeu le matériel suivant (Fig.2 et 3 )

- accéléromètres enregistrant la composante radiale du mouvement en divers points du tube "en service", 
- double intégrateur électronique,

- oscilloscope à mémoire déclanché lors de l'impact.

Deux types de mesures sont réalisés :

- Mesures "en accélération", le long du piedroit Sud du tube, c'est à dire le long du piedroit le plus proche du tube en cours d'excavation, afin de rechercher les points d'accélération maximale ;

- Mesures "en déplacement" en divers points du tube en service (parements, couronne, plateforme) afin de définir le mouvement complet du tube durant la vibration.

La vibration enregistrée ne représente celle du site que durant un intervalle de temps qui exclut le retour des vibrations induites par les réflexions sur les faces libres du modẽle; l'analyse du signal n'est donc effectuée que dans cet intervalle, dont I'ouverture est définie à partir de la distance du capteur considéré au bord libre le plus proche.

Les résultats qui suivent correspondent à un "impact de référence" qui décl nche au droit du front de taille dans le tube Nord, une accélération maximale égale à $150 \mathrm{~g}$ (soit 1,5 g transposé au site).

\section{RESULTATS}

I) Mesures d'accélération le long du piedroit

Les résultats sont reportés sur le graphique de la figure 4.

En abgrisses, les cotes sont exprimées en diamètres de tube, l'origine étant prise au droit du front de taille, $\left(z / D_{m}\right)$.

En ordonnées, les accélérations maximales $\gamma$ max, exprimées en \& de l'accélération de référence ro.

L'observation de la courbe conduit aux remarques suivantes :

- Le niveau maximum de la vibration se trouve, non au droit du front de taille, mais légèrement en avant de celui-ci, à une distance sensiblement égale à un diamètre de tube; l'accélération maximale atteint alors une valeur de l'ordre de $175 \%$ de l'accélération de référence. Un résultat similaire a été constaté par SAKURAI et KITAMURA (1977) lors de mesures effectuées sur un ouvrage réel.

- Le pic d'accélération est précédé d'une "zone d'ombre" dans laquelle l'accélération atteint 50 \% de l'accélération de référence.

- Au voisinage du front de taille, l'évolution des accélérations ne peut nullement

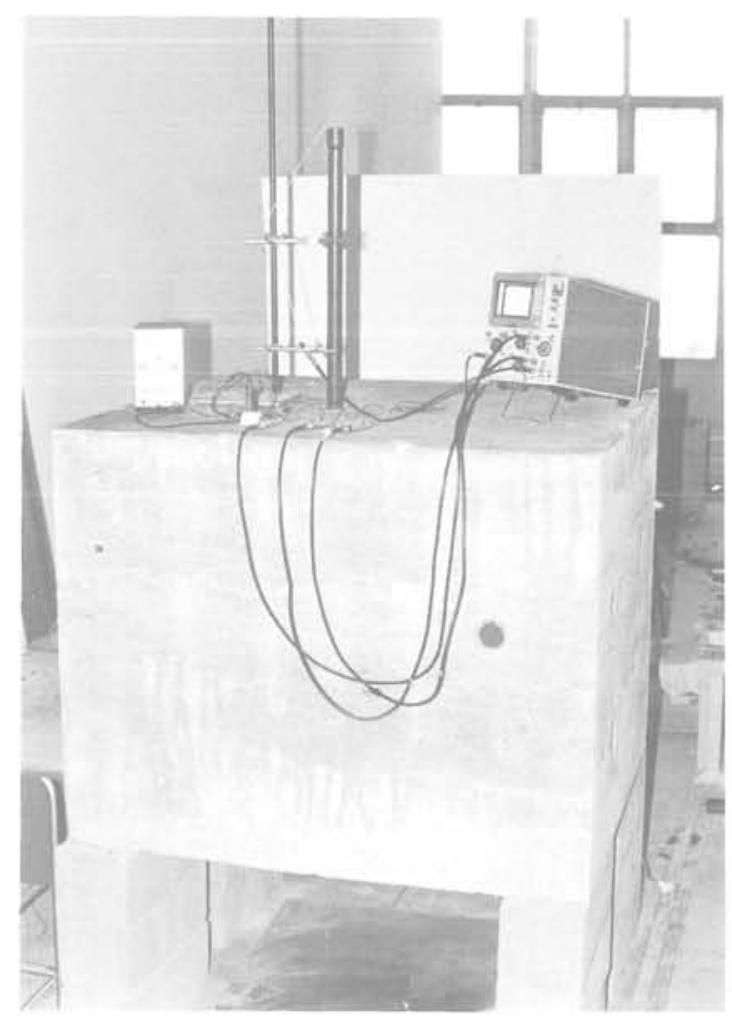

Fig.3 - Vue d'ensemble de la maquette

être représentée par une loi du type $\gamma(z)=\bar{A} z^{-m}$, couramment utilisée en pratique pour des géométries plus simples.

2) Mesure des déplacements, observations sur une section droite

L'enregistrement simultané des vibrations en quatre points d'une section droite permet la reconstitution du "film " des événements durant le passage de la vibration (limité aux temps antérieurs au retour de la réflexion sur les bords du modèle). La figure 5 donne un exemple d'un tel mouvement ; on peut remarquer que, durant la période d'analyse, le mouvement d'une section droite se traduit par une déformation dans laquelle:

- le piedroit situé du côté du tir se déplace environ 10 fois plus que le piedroit opposé ;

- la couronne et la plateforme subissent des déplacements du même ordre de grandeur, environ 5 fois inférieur à celui du piedroit "côté tir".

3) Mesure des déplacements, observations sur une coupe longitudinale

Le mouvement d'ensemble du tube sous l'effet de la vibration est complexe, et difficile à visualiser. A titre d'illustration, on a représenté, sur le graphique de la figure 6, une succession de coupes 
du tube antérieur par un plan horizontal, à douze époques consécutives, de $0,189 \mathrm{~ms}$ à $0,556 \mathrm{~ms}$ après le début de l'impact,permettant ainsi de visualiser la propagation de la vibration. (Une distorsion des échelles des déplacements a été opérée sur les graphiques afin de les rendre plus lisibles : le rapport entre les échelles des déplacements le long des deux piedroits est égal à 5,3).

- La propagation de la vibration le long du tunnel peut être grossièrement décrite par une succession d'élargissements et de rétrécissements, la partie "côté tir" se déplaçant radialement avec une amplitude environ 10 fois supérieure à celle de la partie opposée.

- Le front d'onde se propage avec une vitesse apparente $\mathrm{C}=1460 \mathrm{~m} / \mathrm{s}$. Cette $\mathrm{vi-}$ tesse est voisine de la célérité $C_{T}$ des ondes transversales dans le matériau $\left(\mathrm{C}_{\mathrm{T}}=1560 \mathrm{~m} / \mathrm{s}\right)$, la propagation par ondes longitudinales n'apparait pas, compte tenu de la sensibilité de la chaine de mesure, nécessaire à l'analyse du phénomẽne. L'essentiel de l'énergie vibratoire se propage donc le long du tunnel à une célérité voisine de celle des ondes transversales.

- Un état de phase déterminé, par exemple le maximum d'élargissement du tube, se propage à une célérité apparente nettement

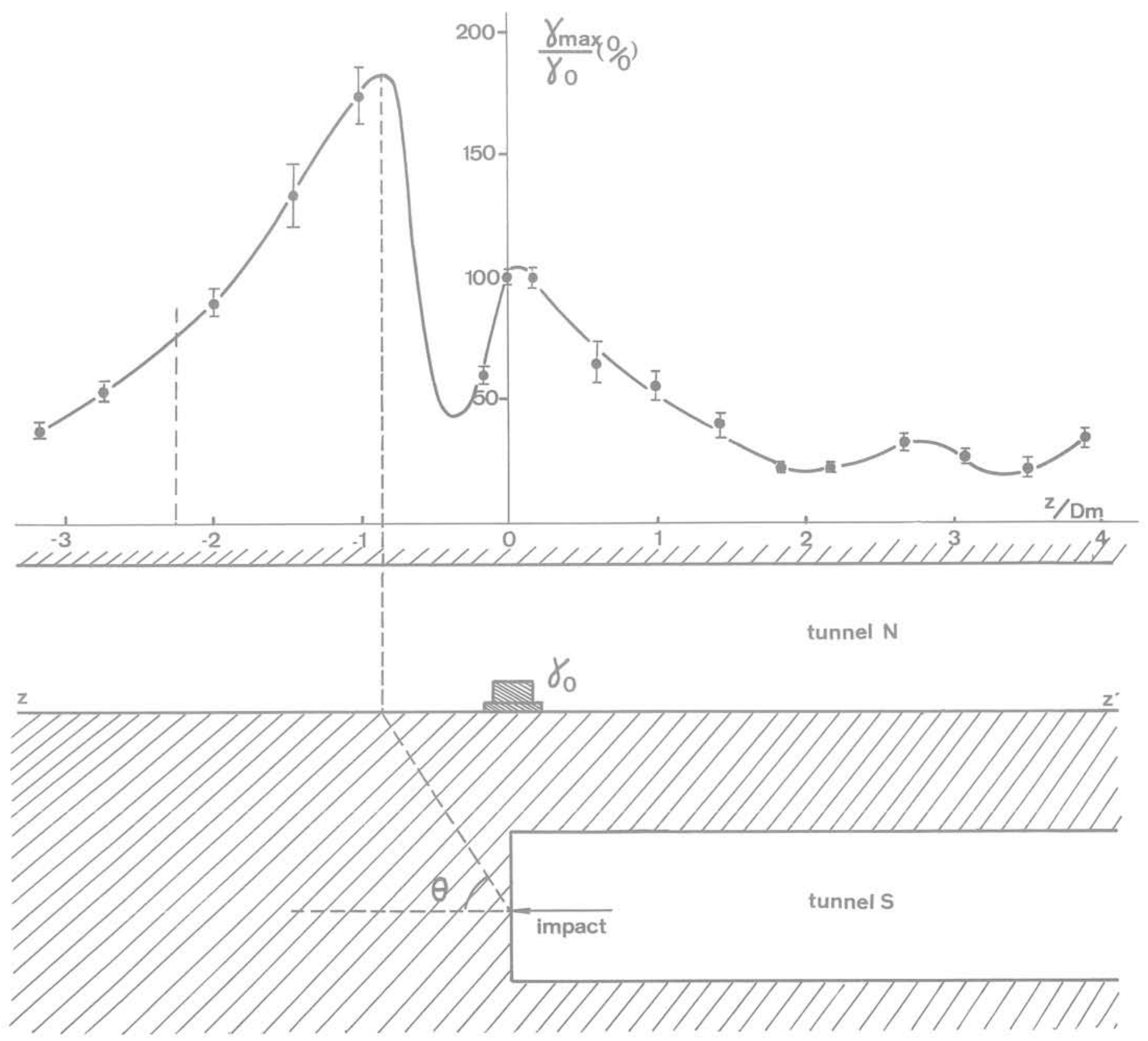

Fig.4 - Evolution de l'accélération maximale $\gamma \max$ le long du piedroit $\mathrm{ZZ}^{\prime}$ 

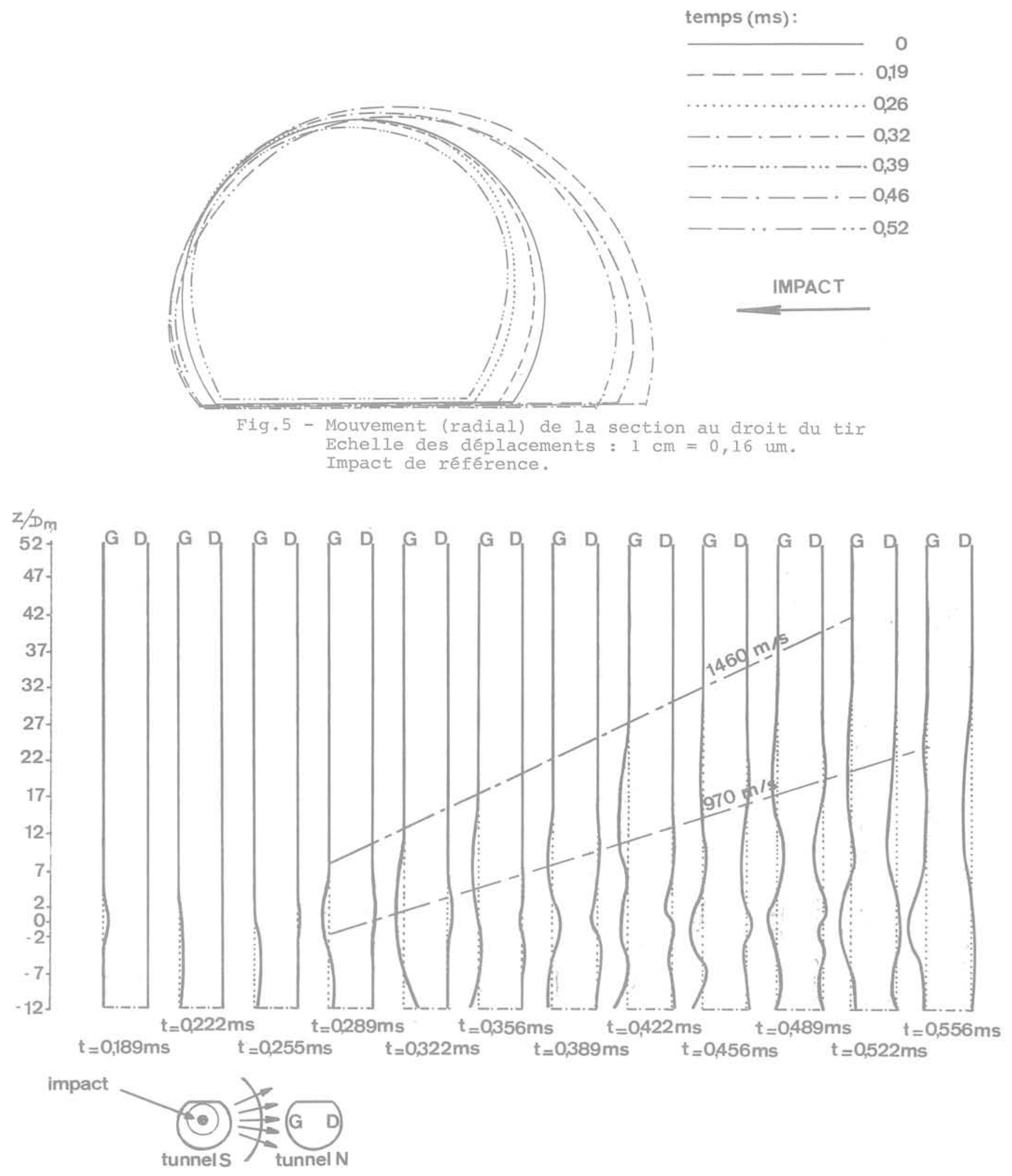

\footnotetext{
Fig.6 - Configuration déformée du tube Nord, coupe au niveau des piedroits. Echelle des déplacements : côté tir : un déplacement égal à un diamètre de tube, lu sur la figure, représente 0,8 um côté opposé : échelle 5,3 fois plus grande.
} 
inférieure, de 1'ordre de 950 à $1000 \mathrm{~m} / \mathrm{s}$.

4) Corrélation avec des essais in situ

A partir de mesures de vibrations, effectuées sur le site du tunnel de Ricard, il a été possible de sélectionner un enregistrement de vibration correspondant à la disposition géométrique de la maquette et des capteurs.

Les caractéristiques de la vibration enregistrée sont les suivantes :

fréquence modale....... $F_{S}=70$ Hertz

déplacement maximum.... $u_{S}=53 \mu \mathrm{m}$

vitesse particulaire... $\mathrm{v}_{\mathrm{S}}=2,35 \mathrm{~cm} / \mathrm{s}$

accélération maximale.. $\gamma_{\mathrm{S}}=1,05 \mathrm{~g}$.

Compte tenu de la fréquence de la vibration engendrée par l'impact sur la maquette et des équations (1) et (2), les caractéristiques homologues sur le modèle sont alors les suivantes :

$$
\begin{aligned}
& F_{m}=6 \cdot 600 \text { Hertz } \\
& u_{m}=56 \cdot 10^{-2} \mu \mathrm{m} \\
& \nabla_{m}=2,35 \mathrm{~cm} / \mathrm{s} \\
& \gamma_{m}=99 \mathrm{~g} .
\end{aligned}
$$

Or l'expérimentation en laboratoire conduit au résultat suivant :

$$
\begin{aligned}
u_{m} & =38 \cdot 10^{-2} \mathrm{um} \\
\text { pour } r_{m} & =99 \mathrm{~g} .
\end{aligned}
$$

Compte tenu de la dispersion qui caractérise les mesures in situ, cet accord semble suffisant et confirme, du moins grossièrement, la validité de l'approche sur modèle expérimental.

\section{CONCLUSION}

1) L'approche par modèle expérimental permet, à condition de respecter les lois de similitude de l'élastodynamique, l'étude en laboratoire d'un problème de propagation de vibration.

2) Il semble possible de simuler les effets vibratoires de l'explosif par impact mécanique et de quantifier l'intensité de la vibration : aínsi l'impact de référence défini précédemment correspond sensiblement, à l'échelle de la maquette, aux tirs de creusement du tube du tunnel de Ricard.

3) Dans cette configuration la vibration du tube antérieur est maximale en un point du piedroit le plus proche du tir, situé à une distance du front de taille égale à un diamètre de tube.
4) L'énerqie vibratoire se propage le long du tube avec une célérité proche de celle des ondes transversales.

5) Le mouvement du tube sous l'effet de la vibration est complexe et se traduit grossièrement par la propagation longitudinale d'une succession d'élargissements et de rétrécissements.

6) Si le modèle proposé permet d'analyser le mouvement de la structure sous l'effet d'un impact, il n'a pas la prétention de représenter la complexité des phénomènes liês à la structure du terrain et au déroulement de la volée d'abattage : l'impact ne peut simuler que le tir des mines tirées au retard zéro mais non celui des mines ultérieures qui composent la salve de la volée.

7) La référence aux lois d'amortissement de la vibration du type $z^{-m}$ ( $z$ : distance au tir) apparait illusoire au voisinage immédiat du front de taille : en particulier, I'effet "axial" du plan de tir se traduit par une dissymétrie de la vibration par rapport au plan du front de taille

\section{REFERENCES}

MANDEL J.

Mécanique des milieux continus, cours de l'Ecole Polytechnique.

SAKURAI S. and KITAMURA Y., (1977)

"Vibration of tunnel due to adjacent blasting operations"

in "Field Mesurements in Rock Mechanics", vol 1, ZURICH

Nous tenons à remercier SCETAUROUTE, Agence du Sud-Est, ISCOTA qui ont participé au financement de cette êtude. 